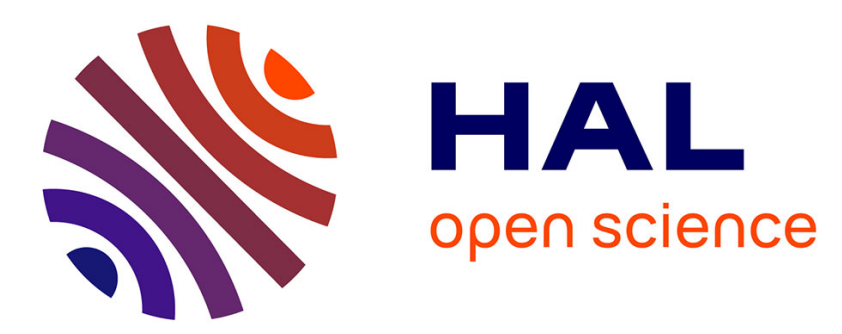

\title{
Condensation of indirect excitons in coupled AlAs/GaAs quantum wells
}

\author{
L. Butov, A. Zrenner, G. Böhm, G. Weimann
}

\section{To cite this version:}

L. Butov, A. Zrenner, G. Böhm, G. Weimann. Condensation of indirect excitons in coupled AlAs/GaAs quantum wells. Journal de Physique IV Proceedings, 1993, 03 (C5), pp.C5-167-C5-170. 10.1051/jp4:1993530 . jpa-00251616

\section{HAL Id: jpa-00251616 https://hal.science/jpa-00251616}

Submitted on 1 Jan 1993

HAL is a multi-disciplinary open access archive for the deposit and dissemination of scientific research documents, whether they are published or not. The documents may come from teaching and research institutions in France or abroad, or from public or private research centers.
L'archive ouverte pluridisciplinaire HAL, est destinée au dépôt et à la diffusion de documents scientifiques de niveau recherche, publiés ou non, émanant des établissements d'enseignement et de recherche français ou étrangers, des laboratoires publics ou privés. 


\title{
Condensation of indirect excitons in coupled AlAs/GaAs quantum wells
}

\author{
L.V. BUTOV, A. ZRENNER, G. BÖHM and G. WEIMANN \\ Walter Schottky Institut, Am Coulombwall, 85748 Garching, Germany
}

\begin{abstract}
The photoluminescence (PL) of excitons confined in an electric field tunable coupled AlAs/GaAs quantum well has been investigated at $\mathrm{T}>350 \mathrm{mK}$ and magnetic field $\mathrm{H}<14 \mathrm{~T}$. In the indirect regime when electrons and holes are separated both in real- and in k-space, magnetic field was found to result in (i) a strong change of both the PL intensity and decay time which is attributed to the anomalies in the exciton transport and (ii) an appearance of a huge broad band noise in the PL intensity which is an evidence of exciton condensation.
\end{abstract}

The electron-hole $(e-h)$ interaction in the neutral $e-h$ system has been predicted to cause the condensation of bound $e-h$ pairs (excitons) into an excitonic insulator state ${ }^{1,2}$. In the case of a dilute excitonic gas $\left(\mathrm{na}_{B}{ }^{d}<1, a_{B}-\right.$ excitonic Bohr radius, $n-e-h$ density, d - dimensionality) the excitons can be considered as rigid Bose - particles and the condensation is analogous to the Bose-Einstein condensation for bosons, while in the case of the dense e-h system ( $\left.\mathrm{na}_{B}{ }^{d} \gg 1\right)$ the condensed state is analogous to the $\mathrm{BCS}$ superconducting state. The transition between those two limits is smooth ${ }^{2}$. The condensation condition can be achieved only if the temperature of the excitons is below a critical temperature $\left(T_{c}\right)$ which turns out to be one of the main difficulties for experimental observation of exciton condensation.

The experimental effort to observe exciton condensation in bulk semiconductors was concentrated mainly on the analysis of the PL lineshape and the transport properties of excitons ${ }^{3-5}$. Degenerate Bose-Einstein statistics (which is a precursor of exciton condensation) have been reported for excitons in $\mathrm{Cu}_{2} \mathrm{O}^{3}$ and in $\mathrm{Ge}^{4}$. Rapid exciton transport observed in $\mathrm{Cu}_{2} \mathrm{O}$ has been attributed to exciton condensation. ${ }^{5}$ In $2 \mathrm{D}$ systems the precursor of the exciton condensation namely the formation of excitons in the dense e-h magnetoplasma has been observed in InGaAs QWs ${ }^{6}$. However it was found that due to the high rate of carrier recombination the electron temperature of photoexcited carriers is higher than $T_{c}{ }^{*} A$ suppression of the recombination rate can be achieved in coupled QWs (CQWs) with separated electrons and holes. CQWs are therefore good candidates for the observation of exciton condensation? 
We have studied the PL of a neutral $e-h$ system in an electric field tunable AlAs/GaAs CQW structure (Fig.1). In this structure electrons and holes are separated both in real- and in $k$-space, the overlap between them can be controlled by an external gate voltage $\left(V_{g}\right)^{8}$. The lifetime in this system is sufficiently long to allow for a thermalization of the indirect excitons down to temperatures below $1 \mathrm{~K}$. Perpendicular magnetic field increases the exciton binding energy and suppresses the kinetic energy of excitons, therefore magnetic field have been applied to improve the critical conditions for exciton condensation. The carriers have been photoexcited by either a cw dye-laser (hw=1.85 eV) or pulsed semiconductor laser ( $\hbar \omega=1.8 \mathrm{eV}, \tau=4 \mathrm{~ns}$ ). $\mathrm{Cw}$ and time resolved $\mathrm{PL}$ have been performed at low temperatures ( $T>350 \mathrm{mK}$ ) at high perpendicular and parallel magnetic fields $(B<14 \mathrm{~T})$.

The direct and indirect excitonic transitions energies are shown in Fig.2 as a function of $\mathrm{V}_{\mathrm{g}}$ for different excitation densities $\left(\mathrm{W}_{\mathrm{ex}}\right)$. In the direct regime $\left(\mathrm{V}_{\mathrm{g}}>0.4 \mathrm{~V}\right)$ both electrons and holes are confined in the GaAs QW. In the indirect regime $\left(V_{g}<0.4 V\right)$ electrons are in the AlAs QW and holes in the GaAs QW. Increasing indirect exciton density results in an increase of $\mathrm{PL}$ energy $\left(\mathrm{E}_{\mathrm{PL}}\right)$. This is due to the net repulsive interaction between indirect excitons. The maximum shift of $E_{\mathrm{PL}}$ for the fixed $\mathrm{w}_{\mathrm{ex}}$ corresponds to the maximum exciton density which is determined by the maximum exciton lifetime. At $\mathrm{V}_{\mathrm{g}}>0$ the exciton lifetime is reduced due to $\Gamma-x$ mixing ${ }^{8}$, while at $V_{g}<-1$ it is reduced due to leakage of carriers through the AlGaAs barriers. The net repulsive interaction between excitons is important as it prevents the condensation into a metallic $e-h$ liquid.

In the indirect regime the integrated PL intensity drops almost two orders of magnitude compared to that in the direct regime. This is due to the fact that in the indirect regime $\tau_{\mathrm{r}} \gg \tau_{\mathrm{nr}}\left(\tau_{\mathrm{r}}\right.$ and $\tau_{\mathrm{nr}}$ are radiative and nonradiative decay times) and the main number of carriers recombine through nonradiative channels. Thus the total recombination lifetime ( $\tau$ ) is determined by $\tau_{\mathrm{nr}}\left(\tau \approx \tau_{\mathrm{nr}}\right)$. $\tau$ was measured to be of the order of $100 \mathrm{~ns}$ (for $V_{g} \approx-0.5 \mathrm{~V}$ ).

The magnetic field was found to result in a strong change of both the PL intensity ( $I_{P L}$ ) and $\tau$ in the indirect regime. This is shown in Fig. 3 for both $B_{1}$ and $B_{\|}$for $V_{g}=-0.4 V$ and $T=350 \mathrm{mK}$. With increasing $B_{\perp}$ both $I_{P L}$ and $\tau$ first increase and then decrease dramatically. $B_{\|}$results in a monotonic increase of $\mathrm{I}_{\mathrm{PL}}$ and $\tau$. The effects disappear at high temperatures $(\mathrm{T}>5 \mathrm{~K})$. The variations of $\mathrm{I}_{\mathrm{PL}}$ and $\tau$ correlate which means that the nonradiative lifetime is subjected to a strong change by magnetic field. Nonradiative Auger recombination in our structures is not efficient since the increase of density does not lead to a reduction of $\tau$. The nonradiative recombination occurs at nonradiative recombination centers (NC). In QWs the low temperature diffusivity of excitons is usually determined by interface roughness scattering and is very low, the rate of nonradiative recombination on NC is determined by the transport of excitons to the $\mathrm{NC}^{9}$. We conclude therefore that the magnetic field has a strong influence on the transport properties of excitons. Increase of $B_{1}$ first leads to a reduction of the exciton diffusivity and then to a strong increase. Increasing $B_{\|}$leads to a monotonic reduction of the diffusivity.

An exciton moving in perpendicular field acquires dipole moment because the Lorenz force acts on electron and hole in the opposite directions. This can lead to increased 
exciton scattering on the interface roughness and thus to the observed reduction of exciton diffusivity. However this mechanism seems to be not able to explain the dramatic increase of exciton diffusivity at high $B_{\perp}$. This behaviour is reminiscent to negative magnetoresistance in electron transport occurring both in the regime of weak ${ }^{10}$ and strong ${ }^{11}$ localization. The magnetic field changes the phases of the charged particles which result in the suppression of coherent backscattering and increase of the electron mobility. As the exciton is composed of two charged particles, the similar effects can be relevant for the exciton transport. However the increase of the exciton diffusivity at high fields is anomalously large and the direct analogy with electron transport is questionable. Also the interaction effects on the transport properties ${ }^{10}$ should be considerably different for excitons due to e-h interaction.

In the indirect regime at perpendicular magnetic fields a huge broad band noise is observed in the PL intensity. The time dependence of the PL signal at $B_{\perp}=11 \mathrm{~T}$ at $\mathrm{V}_{\mathbf{g}}=-0.5 \mathrm{~V}$ is shown in the Fig.4. For comparison the time dependence of $I_{P L}$ at $B=0$ is presented for higher excitation power which corresponds approximately to the same PL intensity. The noise at $B=0$ represents the experimental noise. The power spectrum of the noise shows that it has broad band structure with $\mathrm{S}_{\mathrm{f}} \sim \mathrm{f}^{-0.9}$.

The noise is a strong evidence for the presence of coherence in the exciton system. The noise amplitude is known to be inversely proportional to the number of statistically independent entities in a system ${ }^{12}$. Large noise amplitudes therefore denote that only a small number of entities exists in the macroscopically large photoexcited region.

We believe that the appearance of those macroscopic entities in the exciton system is given by the condensation of indirect excitons. A condensed domain can be considered as one macroscopic entity. The PL signal of condensed excitons is much higher as compared to uncondensed ones ${ }^{13}$. This is mainly due to the fact that the oscillator strength of the excitonic PL is increased with the increase of coherent area ${ }^{14}$ which is given by the domain size in the case of condensed excitons. The formation of condensed domains results therefore in a change of the total PL signal. For the existence of the broad band noise the dephasing time (during which the phase coherence is maintained within a condensed domain) should have a broad distribution ${ }^{15}$.

The conditions under which the noise is observed confirm this interpretation: (i) noise is observed only in the indirect regime for excitons with long lifetime; (ii) noise is observed only at low temperatures. The characteristic temperature dependence of the noise amplitude shows gradual disappearance of the noise between $T=2 \mathrm{~K}$ and $4 \mathrm{~K}$. The decrease of the noise amplitude with increasing temperature reflects a reduction of the average size of condensed domains; (iii) noise is observed at high perpendicular magnetic field which improve condensation conditions.

We would like to thank G.Abstreiter, G.E.W.Bauer, A.B.Dzyubenko, Al.L.Efros, V.D.Kulakovskii for many helpful discussions. L.V.B. thanks FVS foundation for financial support. 


\section{REFERENCES}

[1] L.V.Keldysh, Yu.E.Kopaev, Fiz.Tverd.Tel. 6,2791 (1964), [Sov.Phys.Sol.State 6,2219,1965]

[2] C.Comte and P.Nozieres, J.Phys., 43, 1069 (1982).

[3] D.Hulin, A.Mysyrowicz, and C.Benoît à la Guillaume, Phys.Rev.Lett. 45, 1970 (1980).

[4] V.B.Timofeev, V.D.Kulakovskii, I.V.Kukushkin, Physica 117\&118, 327 (1983).

[5] D.W.Snoke, J.P.Wolfe, A.Mysyrowicz, Phys.Rev.Lett. 64, 2543 (1990).

[6] L.V.Butov, V.D.Kulakovskii,G.E.W.Bauer,A.Forchel,D.Grüzmacher,Phys.Rev.B 46,12765 (1992)

[7] T.Fukuzawa, E.E.Mendez, J.M.Hong, Phys.Rev.Lett. 64, 3066 (1990).

[8] A.Zrenner, P.Leeb, J.Schäfer, G.Böhm, G.Weimann, J.M.Worlock, L.T.Florez, J.P.Harbison, Surf.Sci. 263, 496 (1992).

[9] V.N.Abakumov, V.I.Perel, I.N.Yassievich, North-Holland, Amsterdam,Oxford,NY,Tokyo, 1991.

[10] P.A.Lee, T.V.Ramakrishnan, Rev.Mod.Phys, 57, 287 (1985).

[11] U.Sivan, O.Entin-Wohlman, Y.Imry, Phys.Rev.Lett. 60, 1566 (1988).

[12] B.L.Altshuler, P.A.Lee, R.A.Webb, North-Holland, Amsterdam, Oxford, NY, Tokyo, 1991.

[13] G.E.W.Bauer, Physica Scripta T45, 154 (1992).

[14] J.Feldmann, G.Peter, E.O.Göbel, P.Dawson, K.Moore, C.Foxon, R.J.Elliot, Phys.Rev. Lett. 59, 2337 (1987).

[15] P.Dutta, P.M.Horn, Rev.Mod.Phys. 53, 497 (1981).

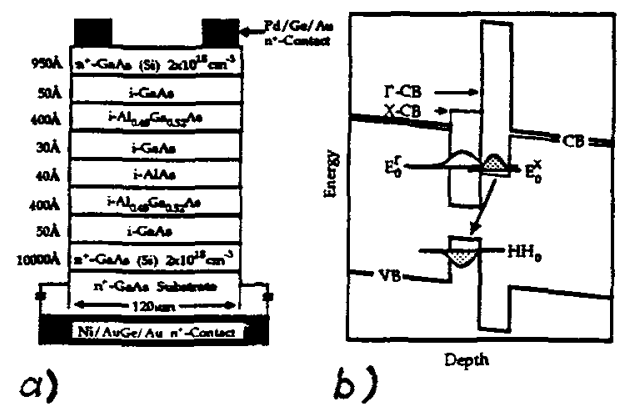

Fig.1 a) Diagram of the $\mathrm{n}^{+}-\mathrm{i}-\mathrm{n}^{+}$structure. b) Band diagram of the real- and k-space indirect AlAs/GaAs structure for negative $\mathrm{V}_{\mathbf{g}}$.

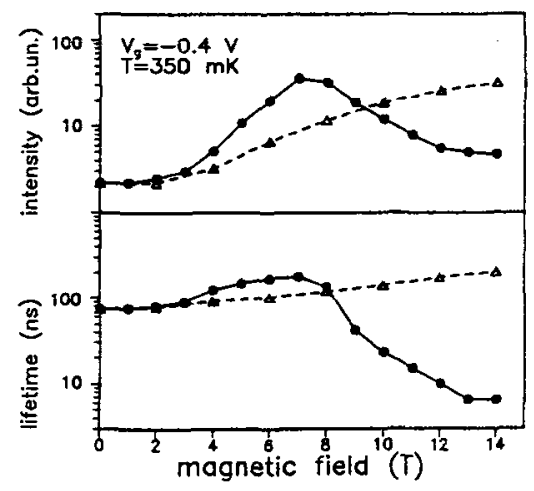

Fig.3 Photoluminescence intensity and decay time in the indirect regime $\left(V_{g}=-0.4 \mathrm{~V}\right)$ at $\mathrm{T}=350 \mathrm{mK}$ versus perpendicular (points) and parallel (triangles) magnetic field.

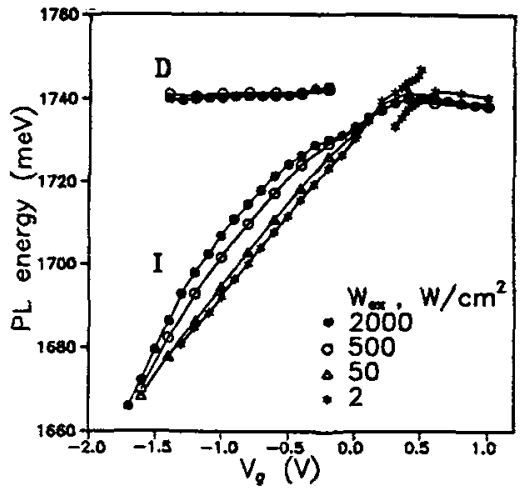

Fig.2 Gate voltage dependences of the direct (D) and indirect (I) transitions at $T=350 \mathrm{mK}$ for different excitation powers.

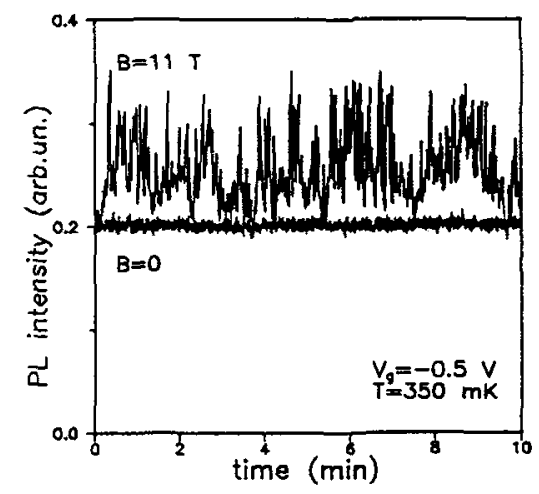

Fig.4 Time dependence of the PL signal in the indirect regime $\left(V_{g}=-0.5 \mathrm{~V}\right)$ at $\mathrm{T}=350$ $\mathrm{mK}$ at $B_{\perp}=11 \mathrm{~T}$, and at $B=0$. $W_{e x}$ for $B=0$ is 6 times higher than that for $B_{\perp}=11 \mathrm{~T}$. 